\title{
How to Share Prosocial Behavior without Being Considered a Braggart?
}

\begin{abstract}
When people share their prosocial behavior on social media, they always face the braggart's dilemma. By sharing their good deeds, they run the risk of being considered braggarts and thus less likable; by staying silent, they receive no credit for what they do. This study proposes a framing strategy to alleviate this concern. By acknowledging a third party involved in the prosocial activity (e.g., organizer or sponsor), one will be perceived as more likable through reducing the suspicion of self-promoting and perceived to have put in more effort. An empirical study based on Twitter data was conducted to confirm our prediction. An experimental study follows to verify the mechanism. The findings provide implications for various stakeholders that take part in prosocial activities.
\end{abstract}

"If you're like most people, self-promotion does not come easily...That being said, we have no issue whatsoever promoting our friends and colleagues."

-Forbes [17]

\section{Introduction}

With the prevalence of social media, people establish their digital identity by sharing their activities and thoughts on social networking sites. Some people actively promote themselves by announcing their prosocial behavior such as donating and volunteering. Most others, however, stay silent despite their desire to share their good deeds [17]. This is caused by the braggart's dilemma - by sharing prosocial behavior, one runs the risk of being considered a braggart and less likable; by staying silent, one runs the risk of receiving no credit for his prosocial effort [3]. Specifically, to be perceived preferably by others, one's prosocial behavior should be based on selflessness. However, the behavior of announcing one's prosocial behavior indicates reputational motivation which is based on selfishness. As a result, self-promoting becomes self-defeating. This dilemma has prohibited social media from reaching its full potential in promoting valuable causes, spreading worthy volunteering opportunities, and most importantly, communicating the norm of helping others [21].
The braggart's dilemma is not only faced by individuals but also by corporations. Corporate social responsibility (CSR) has become an important component of firms' strategy to maximize long-term profit while improving the well-being of society. One example of corporate philanthropic activity is corporate sponsorship for charity runs where corporate sponsors contribute to charities if participants complete a running challenge [27]. However, when companies promote themselves for their good deeds, consumers may perceive their CSR activities to be profit-driven instead of social-welfare-driven. This leads to a lower sales performance for the CSR-oriented company [29]. As an example, Budweiser spent $\$ 5$ million on a Super Bowl commercial in 2017 to brag about donating \$200k worth of water to communities hit by hurricanes, flooding or wildfires. Instead of gaining a reputation as a socially responsible corporation, Budweiser's public image was rendered as profit-driven [20].

Our study proposes a framing strategy that can be used to attenuate the braggart's dilemma:

We propose that individuals will be perceived as more likable when announcing their prosocial behavior with the acknowledgement to a third party than without.

This third party can be the nonprofit organization that organizes the prosocial activity or a corporate sponsor that supports this activity. For example, an individual participant of a charity run can include a thank-you note to the corporate sponsor in his post on social media that announces his participation. That way, this individual shifts the audience's attention from his bragging behavior to the good deed itself, reducing his suspicion as a braggart. In the meantime, he becomes the wingman of the corporate sponsor [15]. This individual helped to promote the corporate sponsor without jeopardizing its public image as a socially responsible corporation. It is a win-win situation for both parties.

Two studies were conducted to examine this proposal. The first study leverages an exogenous change in users' tweets to announce their participation in corporate-sponsored charity exercises. The research context is a mobile distance tracking application that facilitates charity exercises by connecting users and corporate sponsors. For every mile a user runs, walks, or bikes, a matching corporation will donate a fixed amount of money to a nonprofit organization. The 
application encourages users to share their participation on social media and provides a template to ease the sharing process. An example post is "I biked 19.251 miles for@EveryMomCounts.”After 12/11/2013 ${ }^{1}$, the mobile platform changed the post template to include acknowledgment to sponsors. The new post reads "I biked 19.251 miles for @EveryMomCounts. Thx2 @GNC for sponsoring me!" The only change is the addition of corporate mention. This design change provides us with an identifying source to investigate our proposed strategy. To draw a causal inference, we used users' tweets not associated with this activity as a baseline. We found positive evidence that the new template garners more Twitter "likes" than the old one, where Twitter likes are used as a proxy for one's likability.

To investigate the underlying mechanism, we conducted a second study in a lab setting. Subjects recruited from Amazon Mechanical Turk were faced with either version of the tweet (i.e., with or without the acknowledgement to sponsors). They were asked to indicate their likelihood to like the tweet and then to measure the perceived self-promoting tendency as well as the perceived effort level. We find that, consistent with our empirical finding, the template with acknowledgement receives more likes. Perceived selfpromoting tendencies and effort levels are both significant mediators for the link from the treatment to the likability. The tweet with acknowledgement corresponds to a lower self-promoting tendency and a higher likability; it also corresponds to a higher perceived effort level, hence a higher likability.

Our work contributes to the literature of psychology, information systems, and management. Past psychological studies have identified evidence of the braggart's dilemma $[3,11,24]$ and the prominent role of a wingman to promote another party [4]. To our knowledge, it was never previously examined how one can reduce the suspicion as a braggart by being a wingman for another party. This is a novel proposal that can be applied in many circumstances. In the context of information systems, our finding is closely related to user engagement. Our proposal suggests better automatic message designs to encourage voluntary contribution sharing on social media. Such selfreporting content not only raises awareness of the activity but also recruits new users to participate. Our finding also sheds light on the design of CSR campaigns. To recruit wingmen, corporations should actively engage users in their CSR activities and

\footnotetext{
1 This template change was implemented for a short period of time during August 2013 prior to the permanent change on November 2013 Since the temporal change in August 2013 only affects a portion of
}

incentivize them to acknowledge the contribution of the companies.

\section{Theoretical Development}

\subsection{Literature Review}

The braggart's dilemma was studied in both psychology and marketing literature. Berman, Levine [3] found that bragging about one's prosocial behavior signals one's altruism when the prosocial behavior is unknown; it signals one's desire for credit when the prosocial behavior is already known. We use this finding in our experimental design when setting up our research context, as detailed later. Scopelliti, Loewenstein [24] found that people often brag about themselves with the prediction that others will be proud of them, but the reality is disappointing. Others often feel annoyed by the bragging behavior, especially friends. Our work contributes to this finding by proposing a way to reduce such negative impact from prosocial behavior announcements. Vonk [28] argues that people are more likely to brag about themselves when their acts cannot be verified. All these studies focus on when people brag and how their bragging behavior is perceived. Our work differs from their effort by proposing a framing strategy to change others' perception without inhibiting information disclosure.

Message framings are studied in information systems literature to encourage content generation and sharing. Huang, Chen [13] conducted a field experiment to find that users' social sharing is encouraged by monetary incentive as well as relational and cognitive capital framings. Huang, Burtch [12] studied novel message framings based on performance feedback and disentangled the moderation role of gender. Our study differs from these works by directly looking at the likability of different framings from an audience perspective. We also have a focus on user-generated content to communicate their positive attributes. This type of content is different from knowledge-based content that will benefit others with its informational value.

\subsection{Hypothesis Development}

People who show approval and appreciation to others are usually perceived as less egocentric. In sharing prosocial behavior, acknowledging a third party shifts audience's attention from the content generator to the

templates, we choose to study the permanent change in November 2013, which affects all post templates. 
other party or the activity itself. As a result, the content generator is less suspicious of having self-promoting motives. Self-promoting behavior such as sharing one's prosocial activity and achievements is widely studied in psychology literature $[16,22]$. People convey their positive attributes in the hope that others will feel happy for or proud of them. However, others are likely annoyed by such bragging behavior [24]. The reasons are twofold. First, modesty is considered an important virtue is most cultures [30]. Second, self-promoting often leads to social comparison, which causes psychological discomfort for the recipient of such information [11]. This issue becomes worse when a person brags about his prosocial behavior. To be perceived as more likable, one's prosocial behavior needs to be based on selflessness. However, selfpromoting is based on the furtherance of one's selfinterest, making bragging self-defeating [3]. As a result, acknowledging a third-party may increase one's likability by reducing his suspicion as a braggart.

The acknowledgment of a third party may also change the perceived effort level of the focal person. On the one hand, such acknowledgement may lower the perceived effort level of the focal individual because a third party is sharing the credit. Intuitively, this third party may steal the thunder of the focal person as the main contributor. On the other hand, such acknowledgement may render this prosocial activity as a collaborative fundraising effort. The focal person may be perceived to have put more effort in working with other parties to complete the task collectively. Regarding effort, past studies show that altruistic individuals are more likely to expend effort in prosocial behavior because they gain utility from both the wellbeing of beneficiaries and better public images as altruists $[1,2]$. For example, labor productivity is lower in paying jobs than in social jobs [9]. From an attribution perspective, it is widely observed how people use donations to signal their generosity [14]. As a result, a higher effort will lead to better likability. In terms of fundraising, labor-intensive charitable fundraisers are more likely to be successful because hard work and endurance adds positive meanings to the fundraising behavior [19]. Thus, a higher perceived effort level in prosocial activities predicts a higher likability.

The above discussion shows that acknowledging a third party can increase one's likability by reducing his suspicion as a self-promotor and signaling a higher effort level. However, the third party may also steal the thunder and decrease the perceived effort level of the focal individual, leading to a lower likability. We propose the opposing hypotheses below to be examined by our empirical study and lab experiment.
H1: Acknowledging a third party in one's announcement of a good deed increases one's likability.

H2: Acknowledging a third party in one's announcement of a good deed decreases one's likability.

\section{Empirical Analyses}

\subsection{Context}

We collected social media data generated from a distance tracker mobile application - Charity Miles. Charity Miles is a for-profit company founded in 2012 by Gene Gurkoff. It aims to help companies to spend their advertising budget in a revolutionary way. According to Gurkoff, "In most cause marketing arrangements, the company gives a bit to charity and spends 7 to 10 times promoting it. The promotion is what drives the return on investment, not the charity. We are trying to reverse that ratio and generate the marketing R.O.I. that companies want from ordinary advertising. This enables them to repurpose their digital media budgets - money that never ever would have gone to charity - for social good [23]." When users open the app, they will be asked to choose a charity to support. Over 40 causes or charities partner with the app, including Feeding America, Stand Up to Cancer, Autism Speaks, Wounded Warrior Project, and so on. As users walk, run, or bike, the app tracks the distance. Cyclists earn 10 cents while runners and walkers earn 25 cents to the charity of their choice for every mile they complete. According to Skwarecki [26], Charity Miles takes a $50 \%$ cut, which means that for every dollar that goes to charities, the same amount goes to the firm. Their corporate partners include Timex, Johnson \& Johnson, Brooks Running, and others [7].

The app encourages users to share their contributions through social media and developed a post template to ease the sharing process. The post includes the type of activity, the number of miles, and the charity. An example is "I biked 19.251 @ CharityMiles for @EveryMomCounts." On 11/11/2013, the app permanently changed the post template to include the identity of sponsors by adding the message "Thx2 @GNC for sponsoring me!" Below in Figure 1, we show the number of posts that include (solid line) and do not include (dashed line) the keyword "Thx" and "Thank." As shown, posts generated before 11/11/2013 
don't contain these keywords, and posts generated after $11 / 11 / 2013$ have those keywords ${ }^{2}$.

\section{Figure 1: Policy Change in Template Design}

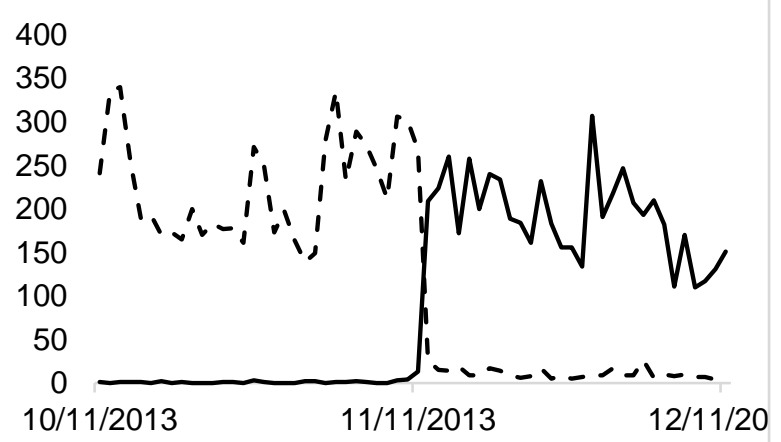

\subsection{Data and Method}

To study the impact of the template change on the social reaction of Twitter likes, we conducted a difference-in-difference (DiD) analysis. The DiD estimator is widely used to account for temporal trends and to analyze policy impact. We modified the classic DiD setting to accommodate our research context. We let individuals' Charity Miles tweets ${ }^{3}$ be the treatment group and their ordinary tweets not associated with Charity Miles be the control group. The dummy variable CharityMiles takes the value of 1 for tweets including "@CharityMiles" and 0 otherwise. The first period is the month before the policy change (10/11/2013 $11 / 11 / 2013)$ and the second period is the month after the policy change (11/12/2013-12/11/2013). A dummy variable After is used to distinguish between these two periods. The treatment group was exposed to the policy change in the second period, where a message like "Thanks to@GNC for sponsoring me!" was added to the original post template. The difference between our setup and a classic DiD estimator is that we separated the groups based on tweets instead of people. As a consequence, we have a two-level structure that results in correlation of tweets from the same individual. To handle such individual-level effects, we added individual-level fixed effects in the model. Since tweets in the treatment and control group contain different content, we further account for tweet-specific attributes using text-mining techniques. In our model, the tweet content is controlled with a set of eight emotion indicators and the length of the content. The emotions are coded using the NRC Word-Emotion Association Lexicon algorithm, which is commonly used to analyze

\footnotetext{
${ }^{2}$ Few posts contain the keywords "Thank" even before the policy change and some posts without the keyword exist after the policy change. This is because the chart shows the raw data collected with
}

the sentiment of tweets [18]. We note that the user-level fixed effects are used to control for time-invariant unobservable features, and the inclusion of the control group accounts for time-varying attributes like users' increasing or decreasing interaction level with their followers.

Other controls include the number of days since the user's last post (DaysSinceLast) and the number of tweets posted the last day users tweeted (NumTweetsLast). Weekly dummies are included in our model to account for any time effect. Basically, we are comparing the difference in tweet likes for ordinary tweets to the difference in tweet likes for CharityMiles tweets over the two periods to reveal the causal influence of the template change. A linear model is used for this analysis where the number of Twitter likes (Like) is our dependent variable. The result stays the same if we use a count regression model. We use $i$ to denote individuals and $j$ to denote tweets. Our model can be specified as:

$$
\begin{array}{r}
\text { Like }_{i j}=\beta_{0}+\beta_{1} \text { After }+ \\
\text { Charity }_{i j}+\mathbf{X} \boldsymbol{\theta}+\alpha_{i}+\gamma_{t}+\varepsilon_{i j},
\end{array}
$$

where $\mathbf{X} \boldsymbol{\theta}=$

$$
\begin{aligned}
& \theta_{1} \text { Anger }_{i j}+\theta_{2} \text { Anticipation }_{i j}+\theta_{3} \text { Disgust }_{i j}+ \\
& \theta_{4} \text { Fear }_{i j}+\theta_{5} \text { Joy }_{i j}+\theta_{6} \text { Sadness }_{i j}+ \\
& \theta_{7} \text { Surprise }_{i j}+\theta_{8} \text { Trust }_{i j}+ \\
& \theta_{9} \text { DaysSinceLast }_{i j}+\theta_{10} \text { NumTweetsLast }_{i j}+ \\
& \theta_{11} \text { ContentLength }_{i j},
\end{aligned}
$$

$\alpha_{i}$ denotes individual-level fixed effects like users' social capital and their popularity on the social networking site. $\gamma_{t}$ represents time effects. In the remainder of the manuscript, the subscriptions are omitted from the variables for brevity.

We collected all tweets containing the keyword “@CharityMiles," the creator's ID, and the number of likes for each tweet. If a tweet did not follow the standard template by reporting miles, activity, and charity, we removed it from our study. Most tweets associated with Charity Miles followed the template though. In total, we obtained 11,084 tweets from one month before and one month after the template change. For users who generated these posts, we collected their other tweets not associated with Charity Miles over the same two-month period. In total, 54,158 other tweets were collected. The summary statistics are reported in Table 1. It is notable that the average number of likes is only 0.5 , indicating that many tweets don't receive any likes. The dummy variable CharityMiles has a mean of

the keyword "CharityMiles." A small portion of these tweets do not follow the template and will be removed in later analyses.

${ }^{3}$ We collected data from Twitter, and we will use tweets to denote posts in the following manuscript. 
0.17 , showing that $17 \%$ of the tweets are related to Charity Miles, and most other tweets are not associated with this topic. From the mean of After, we learn that about $10 \%$ more tweets are generated after the policy change.

Table 1: Empirical Data Statistics

\begin{tabular}{|c|c|c|c|c|}
\hline & mean & sd & $\min$ & $\max$ \\
\hline Like & 0.501 & 1.269 & 0 & 37 \\
\hline CharityMiles & 0.170 & 0.376 & 0 & 1 \\
\hline After & 0.629 & 0.483 & 0 & 1 \\
\hline DaysSinceLast & 2.269 & 3.741 & 1 & 56 \\
\hline NumTweetsLast & 3.311 & 4.658 & 1 & 100 \\
\hline ContentLength & 105.975 & 47.052 & 2 & 4778 \\
\hline Anger & 0.146 & 0.398 & 0 & 4 \\
\hline Anticipation & 0.404 & 0.683 & 0 & 9 \\
\hline Disgust & 0.111 & 0.346 & 0 & 4 \\
\hline Fear & 0.175 & 0.446 & 0 & 5 \\
\hline Joy & 0.431 & 0.708 & 0 & 8 \\
\hline Sadness & 0.157 & 0.416 & 0 & 6 \\
\hline Surprise & 0.183 & 0.441 & 0 & 4 \\
\hline Trust & 0.380 & 0.659 & 0 & 8 \\
\hline
\end{tabular}

Note: the summary statistics for week dummies are omitted for parsimony.

We further looked at the breakdown of different groups before and after the policy change. We find that tweets that received no likes decreased by more than $6 \%$ for Charity Miles tweets after the policy change, as compared to over $2 \%$ for ordinary tweets. This shows model-free evidence that the policy change increased the number of likes.

\subsection{Results}

The estimation results for the DiD model are presented in Table 2. The impact of the policy is reflected in the coefficient of CharityMiles $\times$ After. We can see that the policy positively affects the number of likes $\left(\beta_{3}=0.0485, \mathrm{p}=0.035\right)$. The significant increase in tweet likes shows a higher likability with acknowledgement of corporate sponsors, supporting $\mathrm{H} 1$ and rejecting $\mathrm{H} 2$. These results stay robust even if we remove all the control variables.

We also learn from Table 2 that tweets generated by the Charity Miles template generally received fewer likes $\left(\beta_{2}=-0.2, \mathrm{p}<0.001\right)$ than ordinary tweets. This is possibly because these tweets are less original. Content of greater length is less likely to be liked $\left(\beta_{4}<0\right.$, pvalues $<0.05$ ), possibly due to the higher effort required to read and comprehend the content. We also observe that content with the emotions of anticipation and fear receives significantly fewer likes.
Table 2: Empirical Results

\begin{tabular}{|l|l|l|}
\hline DV: Like & Coefficients & T-statistics \\
\hline CharityMiles & $-0.200^{\star \star \star}$ & $(-10.22)$ \\
\hline After & 0.0286 & $(0.88)$ \\
\hline CharityMiles $\times$ After & $0.0485^{*}$ & $(2.11)$ \\
\hline Anger & 0.00404 & $(0.29)$ \\
\hline Anticipation & $-0.0192^{\star}$ & $(-2.41)$ \\
\hline Disgust & 0.0054 & $(0.38)$ \\
\hline Fear & $-0.0255^{\star}$ & $(-2.11)$ \\
\hline Joy & 0.0155 & $(1.87)$ \\
\hline Sadness & -0.0106 & $(-0.87)$ \\
\hline Surprise & 0.00486 & $(0.44)$ \\
\hline Trust & 0.00178 & $(0.22)$ \\
\hline DaysSinceLast & 0.00134 & $(1.05)$ \\
\hline NumTweetsLast & -0.000345 & $(-0.29)$ \\
\hline ContentLength & $-0.000401^{\star \star *}$ & $(-4.22)$ \\
\hline Week Dummies & \multicolumn{2}{|c|}{ Yes } \\
\hline Fixed Effects & \multicolumn{2}{|c|}{ Yes } \\
\hline Observations & \multicolumn{2}{|c|}{652} \\
\hline \multicolumn{2}{|c|}{ Note: ${ }^{*} p<0.05,{ }^{* *} p<0.01,{ }^{\star * *} \mathrm{p}<0.001$} \\
\hline
\end{tabular}

\subsection{Extensions}

We conducted two extension studies. In the first extension, we changed the dependent variable to the audience's response of "retweet," a content sharing behavior prevalent in Twitter. In the second extension, we used tweet data one year after the policy change to investigate other factors that may drive the likability of content providers.

3.4.1. Response of Retweet. We find that the policy change does not have a significant impact on the response of retweeting. Past work has shown that people retweet others' high-quality content to obtain reputation from their audience [25]. Therefore, retweeting is not an indicator of likability but rather a confirmation of the content. Since the revelation of corporate sponsor does not add content value, retweets do not increase. We also find that lengthy content leads to fewer likes but more retweets. This is an evidence that liking and retweeting are two separate processes: liking focuses on approval of the content generator and retweeting focuses on confirmation of content value.

3.4.2. Factors Driving Likes. We used 128,306 Charity Miles tweets generated one year after the policy change to understand factors that drive Twitter likes. All these tweets follow the social sharing template, and we are able to code the activity (run, bike, or walk) as well as the number of miles. We further categorized the benefitting nonprofit organizations into education, medical assistance, sports, and societal issues. We find that more Twitter likes are received when people exert a higher effort or engage in a more active activity. Specifically, running has a higher estimated coefficient 
than walking, and walking has a greater impact than biking. The variable of mileage has a significant and positive coefficient, indicating that a longer distance is recognized and reacted to via Twitter likes. This extension is a confirmation of our argument concerning the link between effort and likability. It is also a robustness check to show that users' audiences are paying attention to the content of tweets.

\section{Experimental Analysis}

While the empirical study confirms our proposal with real-world application, we are not satisfied merely ascertaining that the acknowledgment of a corporate sponsor increases the likability of the tweet. We seek to understand the causal pathways through which the acknowledgement exerts its effect. In our hypothesis development, we postulate that perceived selfpromoting tendencies and effort levels may play the role of transitioning the acknowledgement to likability. In this study, we conduct a mediation analysis based on a lab experiment to verify the proposed mechanisms.

Our lab experiment also helps us to eliminate alternative explanations for our empirical study. In the real world, alternative factors can cause the link between the template change and the higher likability. For instance, the mention of a corporate sponsor, usually an influential Twitter account like @GNC, is likely to draw traffic from the Twittersphere. Such traffic may explain the higher number of likes for these tweets. Our lab experiment is robust to such alternative explanations because it is fully controlled-every tweet is exposed to a fixed number of subjects and the potential confounder of traffic no longer exists.

\subsection{Method}

One hundred people from the United States were recruited to participate in an online study in exchange for payment. We conducted a between-subjects design with one treatment and one control group. Participants read the following: "Imagine that you follow a colleague John (@JohnSmith) on Twitter. Other than talking about his life, John sometimes shares his charity run activity on Twitter. Below is an example of John's tweet about a charity run activity. Carefully read his Twitter post, and rate the following statements." 4 The main message of the post would be: "I ran 2.251 @CharityMiles for @RedCross.” The sponsor acknowledgement condition will have an additional

\footnotetext{
${ }^{4}$ It is important to mention in the stimuli that this is not John's first
} time sharing charitable content. According to past studies, people earn message "Thx $2 @$ GNC for sponsoring me!" The images of the stimuli are presented in Figure 2.

\section{Figure 2: Stimuli of the Experiment}

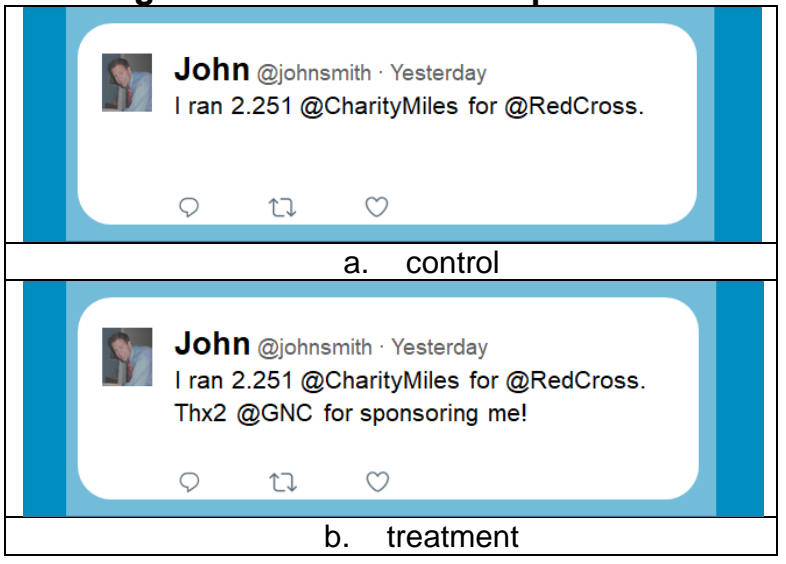

Participants first rated their likelihood to "like" this post on seven-point scales. This measure is the dependent variable. Subjects were then asked about their perceived self-promoting tendency of John and their perceived effort John exerted to complete the charity run. These two measures are our proposed mediators. To gauge the perceived likelihood that John is a self-promoter, we created a five-item self-promotion scale $(\alpha=80.9 \%)$. Specifically, participants rated the following statements on seven-point scales ranging from $1=$ "strongly disagree" to $7=$ "strongly agree": (1) "John likes to show off if he gets the chance," (2) "John likes to impress others," (3) "John likes to be complimented," (4) "John likes to be the center of attention," and (5) "John thinks that he is a special person." This measure for self-promoting tendency was used in Berman, Levine [3]. To examine the perceived effort level, we asked participants to rate the statement that "John exerts a lot of effort in the activity he reported," on seven-point scales. This follows Berman, Levine [3] and Olivola and Shafir [19]. Finally, we included two attention checks. In the first question, participants select whether John contributed to Red Cross or Habitat for Humanity. In the second question, participants select which activity John did from the three options: run, bike, and walk. Three subjects did not answer our questions correctly and were removed from our study. We have 50 subjects in the control group and 47 subjects in the treatment group. The control group has $50 \%$ male subjects, and the average age is 41.04 . The treatment group has $48.9 \%$ male subjects and the average age is 42.48 .

credits for sharing a fundraising activity that is previously unknown to others [3]. 


\subsection{Results}

We conduct a one-way analysis of variance (ANOVA) to determine whether a significant difference exists between the control and treatment groups in terms of likability. We find that the treatment group is more likely to receive Twitter likes than the control group. The coefficient for the treatment dummy has a point estimate of 0.854 , with an $F(1,95)=4.91$ and $p$-value of 0.029. This total effect is reported in the upper panel of Figure 4. We plot the bar chart for the treatment and control group in Figure 3. According to Bartlett's test, the assumption for equal variance is met.

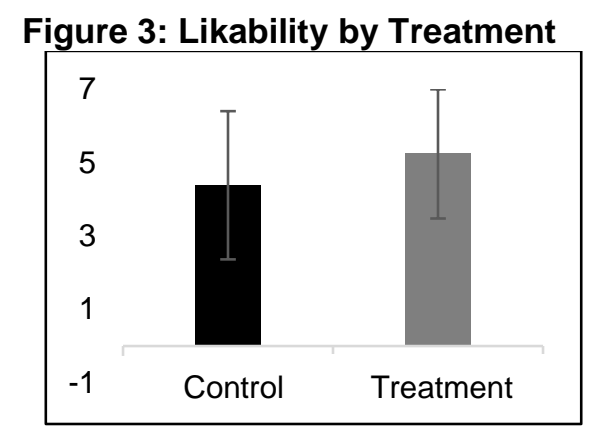

We further conduct a single-step two mediator analysis as shown in the bottom panel of Figure 4. To accommodate two mediators, we run a seemingly unrelated regression equation (SURE) model with three equations [8]. This model allows the error terms of each equation to correlate and is more efficient than separately estimating each equation. The first equation regresses the treatment on the first mediator. The second equation regresses the treatment on the second mediator. The third equation regresses both mediators and the treatment dummy on the dependent variable.

\section{Figure 4: Statistical Diagram}

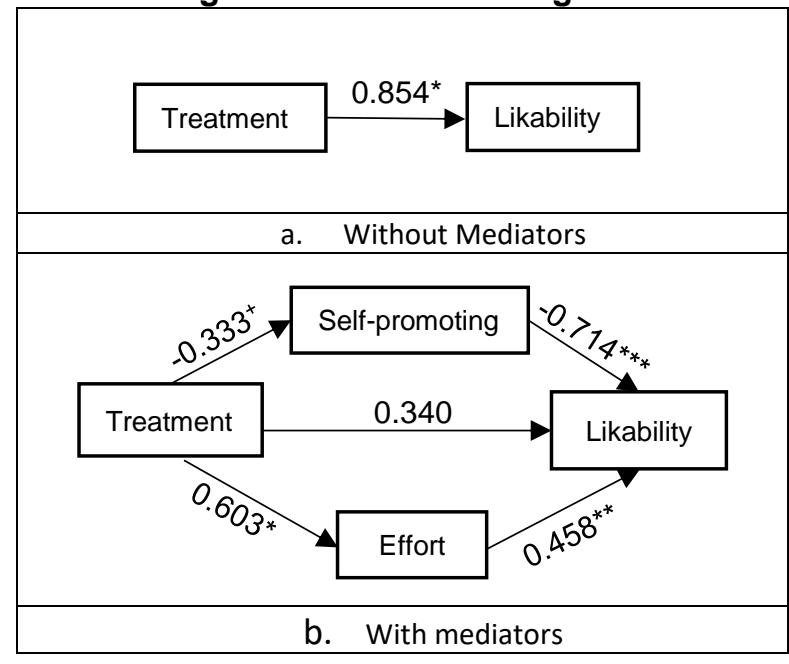

Note: ${ }^{\star} \mathrm{P}<.05 ;{ }^{* \star} \mathrm{P}<.01 ;{ }^{* \star *} \mathrm{P}<.001 ;{ }^{+} \mathrm{P}=0.054$.

The total effect of treatment can be decomposed into the indirect effect of treatment through mediators and the direct effect of treatment. We follow Hayes [8] to calculate these effects and obtain standard errors through bootstrapping. With 5,000 replications, we obtain the results in Table 3. The bias-corrected confidence intervals are all above zero, showing significance for both mediators [8]. Our result shows that $60.19 \%$ of total effect is mediated through our proposed mediators.

Table 3: Experiment Results

\begin{tabular}{|l|l|l|l|l|}
\hline & Coef. & $\begin{array}{l}\text { Boot. } \\
\text { S.E. }\end{array}$ & \multicolumn{2}{|c|}{$95 \%$ Conf. Int. } \\
\hline Ind. Eff. of Self-P. & 0.238 & 0.129 & 0.028 & 0.543 \\
\hline Ind. Eff. of Effort & 0.276 & 0.143 & 0.061 & 0.640 \\
\hline Total Ind. Eff. & 0.514 & 0.184 & 0.205 & 0.939 \\
\hline
\end{tabular}

Note: confidence intervals are bias-corrected.

\section{Discussions}

Our empirical study has shown evidence that including acknowledgement to a third party in one's announcement of prosocial behavior likely leads to more likes on social media. Our experimental study further confirmed this finding and uncovered the mechanism by examining two moderators. Collectively, we show that the mention of a third party reduces one's suspicion of self-promoting while increasing the perception of effort. This is a novel finding that has direct implication to encourage self-presentation of prosocial behavior. While the mediation role of perceived self-promoting tendency is intuitive and consistent with the literature, the mediating role of effort level is not so straightforward. We provide further discussion over the role of perceived effort level.

As we discussed earlier, the acknowledgement to a corporate sponsor may potentially weaken the credit of the individual. How does such a mention of a sponsor strengthen the perceived effort level of this individual? We provide two explanations. First, by acknowledging another party, the focal individual shifts his role from a participant to a social activist. To this end, both his prosocial act and the mention of another party implies their citizenship behavior to make the world better. Therefore, his effort is perceived to be higher because it entails both his participation and promotion for the sponsor. Second, by showing appreciation to the sponsor, the focal individual implies that he is serious about his prosocial contribution. The acknowledgement to sponsors implies that the user is exercising for charities rather than for his self-interest (e.g., fitness or better health). As a result, his perceived effort in 
prosocial behavior is higher. Both alternative explanations can be generalized to other scenarios that involve both individual effort and organizational effort from social organizations, corporate sponsors, and government entities.

\section{Implications}

Our study generates rich theoretical implications. Literature in psychology discussed the importance of presenting one's positive attributes through others rather than self [4]. Intuitively, it is a better idea for one to recruit a wingman to talk about one's good deeds rather than bragging about himself [15]. Our study shows that one can brag about himself while simultaneously being a wingman for others to enjoy higher utility. By acknowledging other parties, one's bragging behavior will become more likable. This unique insight comes from a reverse angle to look at the role of wingman.

Our work also contributes to the literature on usergenerated content. The literature on charitable donations emphasizes the importance of publicizing donations to improve the fundraising performance [21]. Traditionally, donations are announced by organizations during telethons or on the radio and TV. In the age of social media, the social network has been decentralized such that everyone can be an influencer. Therefore, it is critical to understand individual's self-presentation of prosocial behavior. The existing literature in user content generation has covered many motivators including financial incentive, social norms, collectivism, individualism, and social comparison to encourage content generation $[5,12,13]$. To the best of our knowledge, no prior work has considered the negative consequence of content generation. Our work implies that we should not only look at how to increase the benefit of content generation but also how to reduce the cost of content generation.

Our empirical study has implications for the management literature of corporate social responsibility. Traditional CSR activities include causerelated promotions where a portion of product sales is contributed to charities [27]. However, such CSR activities suffer from a low participation rate from individuals. When an individual purchases products with the sales partially going to a charity, it is more of a presentation of the company's altruistic intention rather than the individual's own expression of his social consciousness [6]. As a result, we are unlikely to see people bragging about purchasing such products. A model of Shared Social Responsibility (SSR) was proposed by Gneezy, Gneezy [6] to engage individuals actively by linking their effort to corporate contributions, enabling their self-presentation of social consciousness. The model of Charity Miles is a good example of SSR. For this model to be sustainable, it is important for corporate sponsors to get social exposure. Our work shows that acknowledging corporate sponsors will not dilute the credit of individuals. Rather, it will lead to a win-win situation-the individual will be perceived as more likable and the corporation gains exposure as a socially responsible entity.

Finally, our study is of great practical value to charitable campaign managers both from nonprofit organizations and CSR-oriented companies. To raise awareness and encourage citizenship behavior, fundraising managers usually encourage donors to share their contributions on social media [10]. The design of the system-generated messages to be shared has received limited attention. Our study shows the potential of optimizing such social media post templates (e.g., including corporate sponsors). Further, when CSRoriented companies launch their charitable campaigns, it is important for them to encourage sharing on social media while making sure that they receive credit for their contribution. Johnson \& Johnson has a charitable campaign that donates $\$ 1$ to a charity for every photo users share on their own social media. Along with the photo, the user needs to acknowledge the sponsorship of Johnson \& Johnson. This practice is successful because it highlights both social sharing and acknowledgement of sponsors.

\section{Limitations and Conclusions}

Our study is not without limitations. Unlike psychology works that involve multiple experimental studies with different scenarios to draw a general conclusion, our study is specified in one scenario. This is restricted by our framework that encompasses both empirical and experimental analyses. However, we believe that this work paves a way for future studies to examine the role of showing acknowledgement or appreciation to a third party in user-generated content. By exploring different scenarios and different ways to frame the content, more insights can be generated with respect to the boundary, moderators, and mediators of such a framing strategy. Regarding our empirical study, the major limitation is the lack of controlled manipulation. While the empirical analysis entails an exogenous policy change, such a change applies to all users rather than part of them. We, therefore, cannot provide a straightforward examination of our proposal but have to rely on DiD to account for the time-related confounders. In the meantime, we would like to stress that field experiments concealing sponsors' identify would be very expensive to conduct, and DiD has been widely used to understand policy interventions. 
Despite the above limitations, our work makes a unique contribution to the literature by proposing a novel framing strategy to increase one's likability while sharing his prosocial activities. With that, we conclude our study and look forward to more works in the domain of charitable content generation.

[1] Ariely, D., A. Bracha, and S. Meier, Doing good or doing well? Image motivation and monetary incentives in behaving prosocially. American Economic Review, 2009. 99(1): p. 544-55.

[2] Bénabou, R. and J. Tirole, Incentives and prosocial behavior. American economic review, 2006. 96(5): p. 16521678.

[3] Berman, J.Z., et al., The Braggart's dilemma: On the social rewards and penalties of advertising prosocial behavior. Journal of Marketing Research, 2015. 52(1): p. 90104.

[4] Brandt, A.C., R. Vonk, and A. van Knippenberg, The source effect: Person descriptions by self versus others have differential effects on impression formation. Personality and Social Psychology Bulletin, 2009. 35(7): p. 965-977.

[5] Burtch, G., et al., Stimulating online reviews by combining financial incentives and social norms. Management Science, 2017. 64(5): p. 2065-2082.

[6] Gneezy, A., et al., Shared social responsibility: A field experiment in pay-what-you-want pricing and charitable giving. Science, 2010. 329(5989): p. 325-327.

[7] Gurkoff, G. Who are your Sponsors? 2018 [cited 2018 6/7]; Available from: https://charitymileshelp.zendesk.com/hc/enus/articles/214896108-Who-are-your-Sponsors-.

[8] Hayes, A.F., Beyond Baron and Kenny: Statistical mediation analysis in the new millennium. Communication monographs, 2009. 76(4): p. 408-420.

[9] Heyman, J. and D. Ariely, Effort for payment: A tale of two markets. Psychological science, 2004. 15(11): p. 787793.

[10] Hong, Y., Y. Hu, and G. Burtch, Embeddedness, prosociality, and social influence: Evidence from online crowdfunding. MIS Quarterly, Forthcoming, 2018.

[11] Hoorens, V., et al., The hubris hypothesis: You can self - enhance, but you'd better not show it. Journal of Personality, 2012. 80(5): p. 1237-1274.

[12] Huang, N., et al., Motivating user-generated content with performance feedback: Evidence from randomized field experiments. Management Science, 2018. 65(1): p. 327-345.

\section{References}

[13] Huang, N., et al. Digital Nudging for Online Social Sharing: Evidence from A Randomized Field Experiment. in Proceedings of the 51 st Hawaii International Conference on System Sciences. 2018.

[14] Iredale, W., M. Van Vugt, and R. Dunbar, Showing off in humans: Male generosity as a mating signal. Evolutionary Psychology, 2008. 6(3): p. 147470490800600302.

[15] Jarrett, C. The Braggart's Dilemma: How to Promote Yourself Without Being a Jerk. 2015 [cited 2019 6/5]; Available from: https://99u.adobe.com/articles/51242/thebraggarts-dilemma-how-to-promote-yourself-withoutbeing-a-jerk.

[16] Jones, E.E. and T.S. Pittman, Toward a general theory of strategic self-presentation. Psychological perspectives on the self, 1982. 1(1): p. 231-262.

[17] Marcus, B. Why Taking The Self Out Of Self-Promotion Is The Best Way To Promote Yourself. 2017 [cited 2019 6/5]; Available from: https://www.forbes.com/sites/bonniemarcus/2017/08/30/wh y-taking-the-self-out-of-self-promotion-is-the-best-way-topromote-yourself/\#4836f6ac79d2.

[18] Mohammad, S.M. \# Emotional tweets. in Proceedings of the First Joint Conference on Lexical and Computational Semantics-Volume 1: Proceedings of the main conference and the shared task, and Volume 2: Proceedings of the Sixth International Workshop on Semantic Evaluation. 2012. Association for Computational Linguistics.

[19] Olivola, C.Y. and E. Shafir, The Martyrdom Effect: When pain and effort increase prosocial contributions. Journal of behavioral decision making, 2013. 26(1): p. 91105 .

[20] Orlov, A. Budweiser spent a ton of money on a Super Bowl ad that's all about its charitable efforts. 2018 [cited 2019 6/5]; Available from: https://www.mic.com/articles/187623/budweiser-spent-aton-of-money-on-a-super-bowl-ad-thats-all-about-itscharitable-efforts.

[21] Romano, R. and H. Yildirim, Why charities announce donations: a positive perspective. Journal of Public Economics, 2001. 81(3): p. 423-447.

[22] Rudman, L.A., Self-promotion as a risk factor for women: the costs and benefits of counterstereotypical 
impression management. Journal of personality and social psychology, 1998. 74(3): p. 629.

[23] Schiller, B. This App Pays Your Favorite Charities For Every Mile You Run Or Bike. 2015 [cited 2018 6]; 7]. Available from:

https://www.fastcompany.com/3043144/this-app-pays-

your-favorite-charities-for-every-mile-you-run-or-bike.

[24] Scopelliti, I., G. Loewenstein, and J. Vosgerau, You call it "Self-Exuberance"; I call it "Bragging" miscalibrated predictions of emotional responses to self-promotion. Psychological Science, 2015. 26(6): p. 903-914.

[25] Shi, Z., H. Rui, and A.B. Whinston, Content sharing in a social broadcasting environment: evidence from twitter. Available at SSRN 2341243, 2013.

[26] Skwarecki, B. When Charities Get Donations Because I Clicked, Where Do They Get the Money? 2016 [cited 2018 6/10]; Available from: https://lifehacker.com/whencharities-get-donations-because-i-clicked-where-d1789466184.

[27] Varadarajan, P.R. and A. Menon, Cause-related marketing: A coalignment of marketing strategy and corporate philanthropy. The Journal of Marketing, 1988: p. 58-74.

[28] Vonk, R., Impression formation and impression management: Motives, traits, and likeability inferred from self-promoting and self-deprecating behavior. Social Cognition, 1999. 17(4): p. 390-412.

[29] Wongpitch, S., et al., Effect of corporate social responsibility motives on purchase intention model: An extension. Kasetsart Journal of Social Sciences, 2016. 37(1): p. 30-37.

[30] Ze'ev, A.B., The Virtue of Modesty. American Philosophical Quarterly, 1993. 30: p. 235-46. 